\title{
Isolated Arthroscopic Radial Styloidectomy: The Three-Portal Approach
}

\author{
Guillaume Herzberg, MD, $\mathrm{PhD}^{1,2}$ Marion Burnier, $\mathrm{MD}^{1,2}$ \\ ${ }^{1}$ Wrist Surgery Unit, Clinique Val Ouest, Clinique Parc, I-Trues, Lyon \\ France \\ ${ }^{2}$ Clinique Medipole, I-Trues, Lyon France \\ Address for correspondence Guillaume Herzberg, MD, PhD, 97, \\ Quai Charles de Gaulle, Lyon, France (e-mail: ghmcmo@gmail.com).
}

J Wrist Surg 2020;9:353-356.

Abstract
Keywords
- radial styloidectomy
- scapholunate
advanced collapse
- scaphoid nonunion
advanced collapse
- wrist arthritis
- wrist arthroscopy

Background The current gold-standard technique for radial styloidectomy is arthroscopic. The use of only two dorsal portals may not allow a crystal-clear view of the dorsal arthritic rim of the radius because of the dorsal capsule synovitis.

Objectives To propose a new technique for isolated arthroscopic radial styloidectomy. The addition of a volar radial portal and a sequential procedure are presented. Patients and Methods Two trochars for alternatively viewing from volar radial or 3-4 are used. A 1-2 portal is used for instrumentation with a motorized burr. We have been using the three-portal technique in 34 cases (26 isolated).

Results This modification of the classic arthroscopic radial styloidectomy is technically easy and the view of the dorsal rim of the radius provides a clear definition and treatment of the dorso-radial arthritic pathology.

Conclusions The authors recommend this technical trick to perform an easy and comprehensive isolated palliative or curative arthroscopic radial styloidectomy.
Painful impingement between the radial styloid and the scaphoid may be due to several causes. Radioscaphoid impingement (RSI) may be part of an arthritic wrist collapse regardless of the etiology (scapholunate advanced collapse [SLAC], scaphoid nonunion advanced collapse [SNAC] and articular distal radius malunion involving the radial styloid). Alternatively, RSI may be isolated as a consequence of articular radial styloid malunion or overuse syndrome secondary to sports like karate.

Radial styloidectomy (RS) may thus be indicated either as an isolated procedure (curative to treat an overuse syndrome or palliative for early SLAC or SNAC syndrome in patients unable to sustain a heavier procedure) or as an adjunct during SLAC or SNAC treatment.

Historically, RS has been performed as an open procedure either in conjunction with bone grafting for scaphoid nonunion ${ }^{1}$ or as an isolated procedure through a straight incision centered over the styloid process. ${ }^{2}$ Recommendations regarding the amount of bone to be removed during open RS while preserving the important volar radiocarpal

received

March 23, 2020

accepted

April 28, 2020

published online

June 12,2020 ligaments were provided by Nakamura et al and Siegel and Gelberman. 3,4

In 2005, Yao and Osterman ${ }^{5}$ emphasized the potential advantages of arthroscopic styloidectomy, that is, visualizing exactly how much of the radial styloid, is removed while preserving under direct vision the volar ligaments (especially the origin of the radioscaphocapitate ligament). Arthroscopic radial styloidectomy (ARS) allows decompression of the radial impingement with minimal morbidity. Since then, there has been a limited amount of literature, specifically related to isolated curative or palliative ARS.

The currently described technique for isolated curative of palliative ARS includes the use of two portals, 3-4 and 1-2 or 4-5 and 1-2. . $^{2,9}$

However, when using these techniques, the surgeon may experience difficulties in visualizing the dorsal arthritic rim of the distal radius, as it may be obscured by synovitis.

The purpose of this article was to report a three-portal ARS technique combining the use of a volar radial portal in addition to the classic dorsal 3-4 and 1-2 portals.
Copyright $\odot 2020$ by Thieme Medical Publishers, Inc., 333 Seventh Avenue, New York, NY 10001, USA. Tel: +1(212) 760-0888.
DOI https://doi.org/ 10.1055/s-0040-1712979. ISSN 2163-3916. 


\section{Surgical Technique}

\section{Patient Preparation}

The video screen should be placed into the sagittal plane of the wrist on the opposite side of the table to provide a comfortable view from both the dorsal and volar side of the wrist.

The authors use a shoulder traction system and a counter pressure device just distal to the tourniquet, allowing a stable traction position (4-6 kg) with the entire forearm free in the surgical field. Chinese finger traps are preferred over a traction hand.

\section{Procedure}

We use dry wrist arthroscopy combined with automatic wash outs (AWO) to evacuate the bone debris. ${ }^{10,11}$ We first prepare the three-portal ARS here(-Fig. 1). An outside-in volar radial portal is performed as described previously. ${ }^{12,13}$ A 2-cm longitudinal incision centered on the proximal wrist crease is performed along the flexor carpi radialis (FCR). The FCR is released and slightly retracted ulnarly, so as to expose its floor. A clamp is used to pierce the volar capsule at the level of the proximal palmar crease, to enter the radiocarpal joint between the radioscaphocapitate and long radiolunate ligaments. A blunt trochar follows the same path to be left in place during the whole procedure.

The surgeon moves to the dorsal aspect of the wrist to perform the classic 3-4 and 1-2 portals. A second blunt trochar is placed into the 3-4 portal, to be left in place during the whole procedure. A $3.5 \mathrm{~mm}$ burr is placed into the 1-2 portal and left in this position during the whole procedure.

Once the three portals are prepared, the surgeon follows three sequential steps.
First, the arthroscope is placed into 3-4 and burring of the volar-radial rim of the radial styloid is performed. Combined with preoperative planification, the known diameter of the burr helps in deciding how much bone should be removed. The volar trochar which was previously inserted is directed to the ulnar part of the wrist to move it out of the way. The bony resection should not go beyond the radial border of the radioscaphocapitate ligament.

In a second step, the surgeon moves to the volar aspect of the wrist and the arthroscope is moved into the volar radial portal while the 3-4 dorsal trochar is left in place and directed ulnarwards to stay out of the way. Burring of the dorsoradial arthritic rim of the radial styloid is performed. This is an easier step compared with a two-portal technique since the synovitis does not obscur the view (-Fig. 2).

During the third and final step, the surgeon moves again to the dorsal aspect of the wrist to get a final assessment of the amount of bone which was resected. Peroperative fluoroscopy may be performed anytime if necessary. A soft dressing is applied followed by a splint for 3 weeks. Self-rehabilitation is begun at day 3 .

\section{Patients and Methods}

Between 2006 and 2017, we performed 34 ARS cases at our institution. The ratio of female to male was $1: 33$. Mean age was 46 years (minimum, 22 years and maximum, 69 years). We performed isolated curative ARS in four patients (three overuse osteoarthritis and one articular radial styloid malunion) and isolated palliative ARS in 24 (10 SLAC 2 and 14 SNAC 1). A total of six ARS were combined with another concomitant procedure (one scapholunate dorsal capsulodesis, one

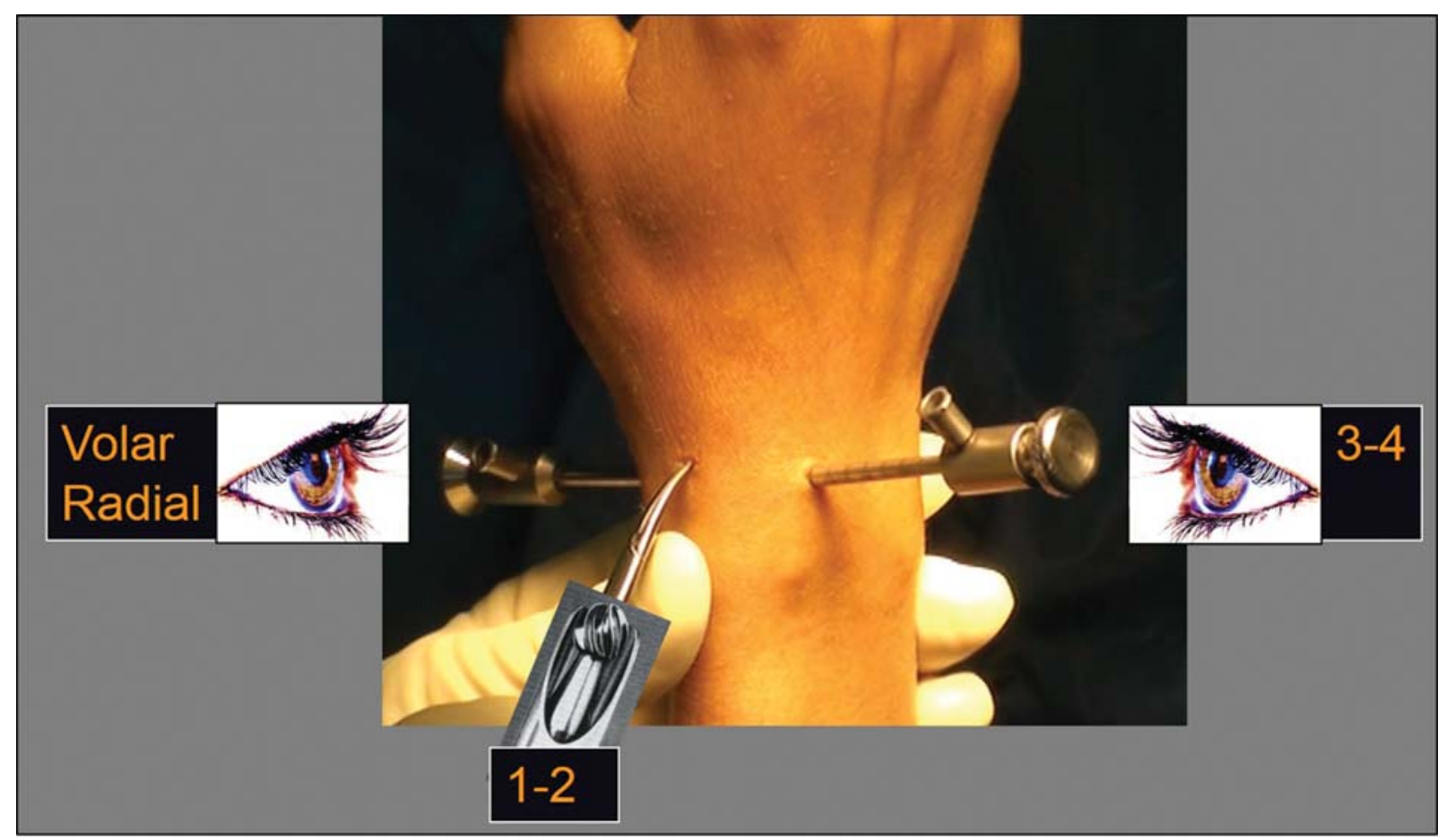

Fig. 1 Three portals technique: A trochar is introduced into the volar radial portal and another in the 3-4 portal. The burr is maintained in the 1-2 portal during all the procedure. 


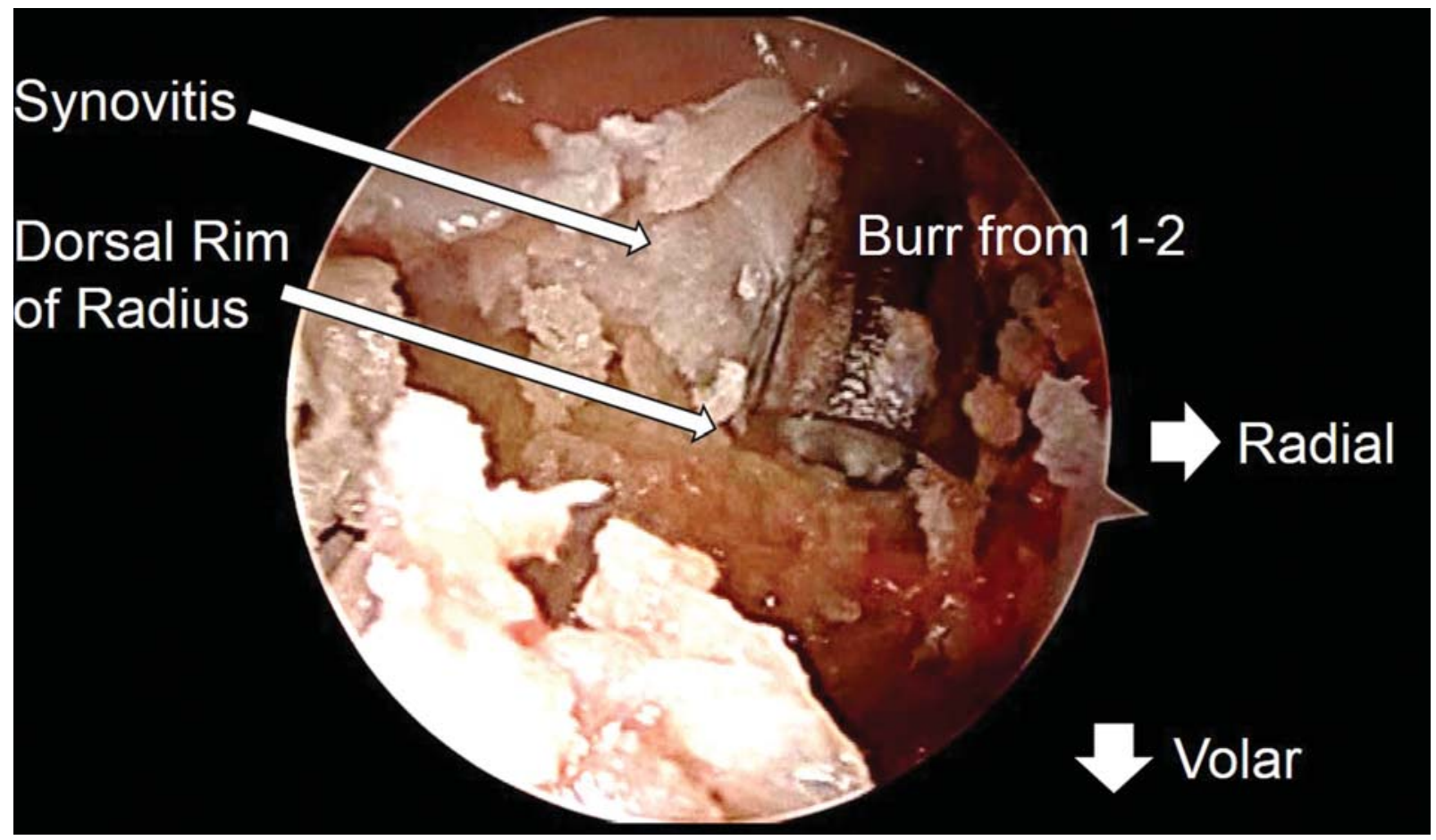

Fig. 2 Intra operative arthroscopic view through the volar radial portal. This allows a better view of the dorsal rim and a better resection of the dorsal part of the radial styloidectomy with the burr in the 1-2 portal.

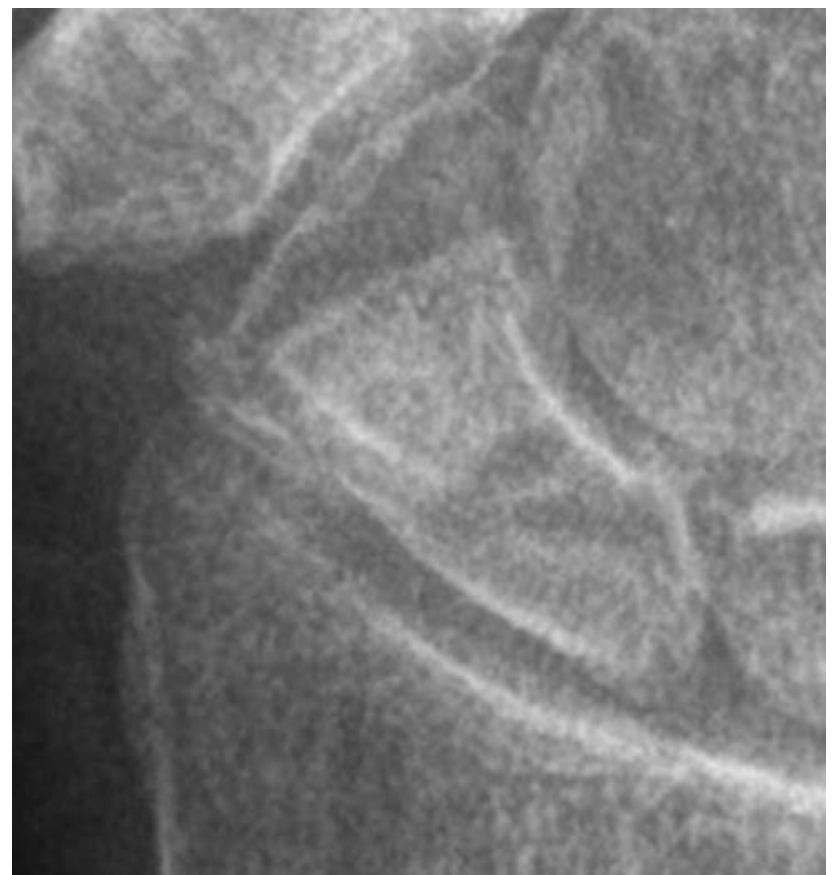

Fig. 3 Preoperative posteroanterior radiograph focusing on the radial styloid impingement.

scaphoid nonunion cancellous bone grafting, one scaphoid proximal pole nonunion KW fixation, and three APSI pyrocarbon implants for necrotic proximal pole nonunion).

Interestingly enough, only one patient in whom an isolated palliative ARS was performed for SLAC wrist sustained a secondary procedure (proximal row carpectomy). All other patients having sustained isolated ARS were satisfied (-Figs. 3 and 4). No complications was reported including

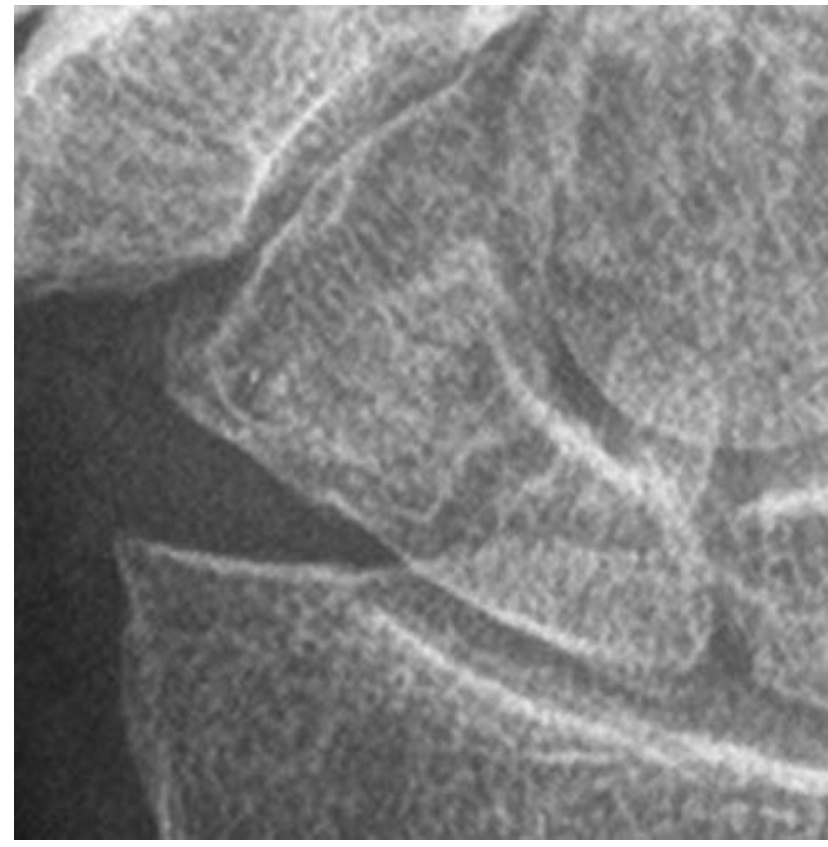

Fig. 4 Postoperative posteroanterior radiograph focusing on the resection of the radial styloid impingement.

no vascular or neurologic damage and no carpal ulnar translation.

\section{Discussion}

Painful impingement between the radial styloid and the scaphoid may be part of an arthritic wrist collapse (SLAC or SNAC) or may be isolated as the result of an overuse 
syndrome (most often from specific sports, like karate) or secondary osteoarthritis.

In patients focusing on dorsoradial trigger wrist pain from one of the aforementioned etiologies, ARS may be indicated. ARS may be performed either as an isolated procedure (curative to treat an overuse syndrome or palliative for early SLAC or SNAC syndrome in patients is unable to sustain a heavier procedure), or as an adjunct during a SLAC or SNAC treatment.

In 2005, Yao and Osterman ${ }^{5}$ emphasized the potential advantages of ARS, that is, visualizing exactly how much of the radial styloid, is removed while preserving under direct vision the volar ligaments (especially the origin of the radioscaphocapitate ligament). ARS allowed decompression of the radial impingement with minimal morbidity. Given the technological and clinical advances in wrist arthroscopy, it became quickly obvious that ARS was by far more appealing than the historical open radial styloidectomy. ${ }^{1}$

Since then, there has been a limited amount of literature related to the technique and results of isolated curative or palliative ARS. The surgical technique used two dorsal portals, 3-4 and $1-2$ or $4-5$ and $1-2 .^{2,6-9}$

Using dorsal two-portal technique, the authors experienced difficulties in visualizing the dorsal arthritic rim of the distal radius where most of the pathology stays since it may be obscured by synovitis from the dorsal capsule. This is why we began in 2006 to use a three-portal technique combining the use of dorsal 3-4, dorsoradial 1-2, and volar radial portals for ARS. The volar radial portal was recently described. ${ }^{14}$ This portal may be performed from inside out using a rigid rod inserted into 3-4 and directed to the interval between the radioscaphocapitate and long-radiolunate ligaments.

It is the authors' opinion that a slight variation of the angulation of the rigid rod may jeopardize either the median nerve or the radial artery. We prefer to use an outside-in technique as described by Slutsky. ${ }^{14}$ To make the procedure as smooth and quick as possible, it is wise to prepare the three portals before beginning the procedure and to prepare first the volar radial portal. The position of the video screen should be carefully assessed before the procedure since the surgeon has to face both the dorsal and volar aspects of the wrist while watching the screen. The main advantage to use the threeportal technique is that the arthroscope placed into the volar radial portal faces directly the dorsal arthritic rim of the radius where most of the pathology is located. This allows a thorough resection of the arthritic radial styloid when an isolated ARS is indicated. Our clinical results are similar to previously published results. ${ }^{8,15}$

With the use of a third volar radial portal and following appropriate sequence, an isolated ARS can be performed routinely and safely. In selected patients with elective dorsoradial wrist associated, ARS is efficient in relieving pain and achieving satisfactory midterm outcomes.

\section{Conflict of Interest}

None declared.

\section{References}

1 Barnard L, Stubbins SG. Styloidectomy of the radius in the surgical treatment of nonunion of the carpal navicular; a preliminary report. J Bone Joint Surg Am 1948;30A(01):98-102

2 Kalainov DM, Cohen MSweet S. Radial styloidectomy. In: Geissler WB, ed. Wrist Arthroscopy. New York, NY: Springer-Verlag; 2005: 134-138

3 Nakamura T, Cooney WP III, Lui WH, et al. Radial styloidectomy: a biomechanical study on stability of the wrist joint. J Hand Surg Am 2001;26(01):85-93

4 Siegel DB, Gelberman RH. Radial styloidectomy: an anatomical study with special reference to radiocarpal intracapsular ligamentous morphology. J Hand Surg Am 1991;16(01):40-44

5 Yao J, Osterman AL. Arthroscopic techniques for wrist arthritis (radial styloidectomy and proximal pole hamate excisions). Hand Clin 2005;21(04):519-526

6 Baratz ME. Radial styloidectomy. In: Baratz ME, Rosenwasser MP, Adams BD, Kozin SH, eds. Wrist Surgery. Tricks of the Trade. New York, NY: Thieme; 2006:128

7 Gill LE, Chhabra AB. The role of arthroscopic evaluation and debridement in wrist arthritis. In: Chhabra $A B$, Isaaca J, eds. Arthritis and Arthroplasty of the Hand, Wrist and Elbow. Philadelphis, PA: Saunders-Elsevier; 2015:15

8 Levadoux M, Cognet JM. [Arthroscopic styloidectomy]. Chir Main 2006;25(Suppl 1):S197-S201

9 Mathoulin C. Arthroscopic radial styloidectomy. In: Wrist Arthroscopy Techniques. Stuttgart, Germany: Thieme; 2015:33-35

10 Del Pinal F. Technical tips for (dry) arthroscopic reduction and internal fixation of DRF. JHSA 2011;36A(10):1694-1705

11 Herzberg G. Perilunate injuries, non dislocated (PLIND). J Wrist Surg 2013;2(04):337-345

12 Slutsky DJ. Volar portals in wrist arthroscopy. J Am Soc Surg Hand 2002;2:225-232

13 Tham S, Coleman S, Gilpin D. An anterior portal for wrist arthroscopy. Anatomical study and case reports. J Hand Surg [Br] 1999;24(04):445-447

14 Slutsky DJ. Wrist arthroscopy through a volar radial portal. Arthroscopy 2002;18(06):624-630

15 Ruch DS, Chang DS, Poehling GG. The arthroscopic treatment of avascular necrosis of the proximal pole following scaphoid nonunion. Arthroscopy 1998;14(07):747-752 